\title{
Resolvable Even-Cycle Systems with a 1-Rotational Automorphism
}

\author{
Miwako Mishima, ${ }^{1}$ Hung-Lin $\mathrm{Fu}^{2}$ \\ ${ }^{1}$ Information and Multimedia Center, Gifu University, 1-1 Yanagido, \\ Gifu 501-1193, Japan, E-mail: miwako@info.gifu-u.ac.jp \\ ${ }^{2}$ Department of Applied Mathematics, National Chiao Tung University, \\ 1001 Ta Hsueh Road, Hsinchu, Taiwan, Republic of China, \\ E-mail: hlfu@math.nctu.edu.tw
}

Received 30 April 2002; revised 23 June 2003

\begin{abstract}
In this article, necessary and sufficient conditions for the existence of a 1rotationally resolvable even-cycle system of $\lambda K_{v}$ are given, which are eventually for the existence of a resolvable even-cycle system of $\lambda \boldsymbol{K}_{\boldsymbol{v}}$ ( () 2003 Wiley Periodicals, Inc. J Combin Designs 11: 394-407, 2003; Published online in Wiley InterScience (www.interscience.wiley.com).

DOI $10.1002 /$ jcd. 10058
\end{abstract}

Keywords: cycle system; extended Skolem sequence; 1-rotationally resolvable

\section{INTRODUCTION}

Let $V$ be the point-set (or the vertex-set) of $\lambda K_{v}$ and $\mathcal{C}$ a collection of cycles of length $m$, called $m$-cycles, whose edges partition the edges of $\lambda K_{v}$. Then the pair $(V, \mathcal{C})$ is called an $m$-cycle system of $\lambda K_{v}$ or a $\lambda$-fold $m$-cycle system of order $v$.

Assume $(V, \mathcal{C})$ to be an $m$-cycle system of $\lambda K_{v}$. In an automorphism group of $(V, \mathcal{C})$, i.e., in a group of permutations on $v$ points leaving the collection $\mathcal{C}$ of cycles invariant, if there is an automorphism $\pi$ of order $v-1$ with a single fixed point, then the system $(V, \mathcal{C})$ is said to be 1-rotational. For a 1-rotational $m$-cycle system of $\lambda K_{v}$, the point-set $V$ can be identified with $\{\infty\} \cup \mathbb{Z}_{v-1}$, i.e., a fixed point $\infty$ and the residue group of integers modulo $v-1$. In this case, the automorphism can be represented by

$$
\pi: \infty \mapsto \infty, i \mapsto i+1(\bmod (v-1)) \quad \text { or } \quad \pi=(\infty)(0,1, \ldots, v-2)
$$

Correspondence to: Miwako Mishima; E-mail: miwako@info.gifu-u.ac.jp

Contract grant sponsor: Ministry of Education, Culture, Sports, Science and Technology (Grant-in-Aid for Young Scientists) (to M.M.); Contract grant numbers: (B) 13780173 and (B) 1570023; Contract grant sponsor: National Science Council of the Republic of China (to H.-L.F.); Contract grant number: NSC-90-2115-M-009-027.

(C) 2003 Wiley Periodicals, Inc. 
acting on the point-set $V=\{\infty\} \cup \mathbb{Z}_{v-1}$. For a cycle $C$ of a 1-rotational $m$-cycle system of $\lambda K_{v},(V, \mathcal{C})$, a cycle orbit of $C$ is defined by $\left\{C+i \mid i \in \mathbb{Z}_{v-1}\right\}$. The length of a cycle orbit is its cardinality. A cycle orbit of length $v-1$ is said to be full, otherwise short. A base cycle of a cycle orbit $\mathcal{O}$ is a cycle $C \in \mathcal{O}$ chosen arbitrarily. Any 1-rotational $m$-cycle system is generated from base cycles. For an $m$-cycle system of $\lambda K_{v},(V, \mathcal{C})$, if the collection $\mathcal{C}$ of cycles can be partitioned into $s(=\lambda(v-1) / 2)$ 2 -factors (in terms of block designs, resolution classes), $R_{1}, \ldots, R_{s}$, then the system $(V, \mathcal{C})$ is said to be resolvable and $\mathcal{R}=\left\{R_{1}, \ldots, R_{s}\right\}$ is called a resolution of the system. Obviously, for the existence of a resolvable $m$-cycle system of $\lambda K_{v}, v$ $\equiv 0(\bmod m)$ and $\lambda(v-1) \equiv 0(\bmod 2)$ must hold. A resolvable $m$-cycle system is said to be 1-rotationally resolvable when it admits $\pi=(\infty)(0,1, \ldots, v-2)$ as an automorphism leaving a resolution invariant as well.

We refer the reader to Refs. [13] and [15] for extensive surveys and bibliographies on cycle systems. For the resolvability, it is known that the existence problem for a resolvable $m$-cycle system of $\lambda K_{v}$ (or $\lambda K_{v}-F$ if $\lambda$ is odd and $v$ is even, where $F$ is a 1 -factor of $K_{v}$ ) is equivalent to the Oberwolfach problem with factors of uniform length (see, for the original definition, [1] or [12]). The spectrum for the Oberwolfach problem with this particular restriction on the length of factors was settled by Alspach et al. [2], Gvozdjak [10], and Hoffman and Shellenberg [11]. For the rotationality only, Buratti [6] got the explicit solution to the existence problem for 1-rotational $m$-cycle systems of $K_{v}$ with $m$ odd for $v<3 m$. However, we do not know much about 1-rotationally resolvable cycle systems. Recently the authors [9] proved through extended Skolem sequences and similar sequences of integers that a 1-rotationally resolvable 4-cycle system of $2 K_{v}$ exists if and only if $v \equiv 0(\bmod 4)$. In this article, we will establish necessary and sufficient conditions for the existence of a 1-rotationally resolvable even-cycle system of $\lambda K_{v}$ in general as the sequel to Ref. [9].

\section{CONVERSION OF THE PROBLEM}

For the existence of a $2 m$-cycle system of $\lambda K_{v}$, it is necessary that $2 m$ divides $\lambda v(v-1) / 2, \lambda(v-1)$ is even, and either $v=1$ or $v \geq 2 m$ (see [15]). By a simple argument on parameters, we have $v \equiv 0(\bmod 2 m)$ and $\lambda \equiv 0(\bmod 2)$ as necessary conditions for the existence of a resolvable $2 m$-cycle system of $\lambda K_{v}$. That allows us to state the following.

Lemma 2.1. Necessary conditions for the existence of a 1-rotationally resolvable $2 m$-cycle system of $\lambda K_{v}$ are that $\lambda$ is even and $v \equiv 0(\bmod 2 m)$.

Our purpose is to prove that the necessary conditions in Lemma 2.1 are also sufficient. Note that if the sufficiency is guaranteed for $\lambda=2$, then it will follow for any even $\lambda$. So, first of all, we will examine the case $\lambda=2$. It should be mentioned that any 1-rotational $2 m$-cycle system of $2 K_{v}$ consists of $v /(2 m)$ full cycle orbits, which implies that $v \equiv 0(\bmod 2 m)$ is a necessary condition also for a $2 m$-cycle system of $2 K_{v}$ to be 1-rotational.

To start with, we will review the definition of an extended Skolem sequence, which is the key to our constructions for 1-rotationally resolvable $2 m$-cycle systems of $2 K_{v}$. 
A $k$-extended Skolem sequence of order $t$, denoted by $k$-ext $\mathcal{S}_{t}$, is a sequence $\left(s_{1}, \ldots, s_{2 t+1}\right)$ of $2 t+1$ integers in which $s_{k}=0$ and for each $j \in\{1, \ldots, t\}$, there exists a unique $i \in\{1, \ldots, 2 t+1\} \backslash\{k\}$ such that $s_{i}=s_{i+j}=j$. A $k$-ext $\mathcal{S}_{t}$ is also represented as a collection of $t$ ordered pairs $\left\{\left(a_{j}, b_{j}\right): 1 \leq j \leq t, b_{j}-a_{j}=j\right\}$ with $\cup_{j=1}^{t}\left\{a_{j}, b_{j}\right\}=\{1, \ldots, 2 t+1\} \backslash\{k\}$. When $k=t+1$, the sequence is often referred to as a Rosa sequence or a split Skolem sequence (see [8] or [16]). It is known due to Rosa [16] that a split Skolem sequence gives a solution to Heffter's second difference problem. In fact, Skolem sequences and their generalizations, including extended Skolem sequences, are fairly useful in forming some other combinatorial structures as evidenced by Refs. [3-5,7,14,17] and others. In Ref. [9], the reader can see how a $(t+1)$-ext $\mathcal{S}_{t}$ and a $t$-ext $\mathcal{S}_{t}$ containing the pair $(t+1,2 t+1)$ (as collections of ordered pairs) work to derive base cycles for 1-rotationally resolvable 4-cycle systems of $2 K_{v}$. That is the basic idea we are going to generalize in order to settle the existence problem of 1-rotationally resolvable $2 m$-cycle systems of $2 K_{v}$ for all $m \geq 2$.

The spectrum of a $k$-ext $\mathcal{S}_{t}$ is known due to Baker [3].

Theorem 2.2 ([3]). There exists a $k$-ext $\mathcal{S}_{t}, 1 \leq k \leq 2 t+1$, if and only if either

(1) $k$ is odd and $t \equiv 0$ or $1(\bmod 4)$; or

(2) $k$ is even and $t \equiv 2$ or $3(\bmod 4)$.

It readily follows from Theorem 2.2 that there exist a $(t+1)$-ext $\mathcal{S}_{t}$ if $t \equiv 0,3(\bmod 4)$ and a $t$-ext $\mathcal{S}_{t}$ if $t \equiv 1,2(\bmod 4)$. In the remainder of the present section, the main constructions for 1-rotationally resolvable $2 m$-cycle systems of $2 K_{v}$ will be presented concerning those two cases of $t$, respectively.

Without loss of generality, let $v=2 m(t+1)$ and thus $V=\{\infty\} \cup \mathbb{Z}_{2 m(t+1)-1}$ for $t \geq 0$. Since any 1 -rotationally resolvable $2 m$-cycle system of $2 K_{v}$ consists of $v /(2 m)$ full cycle orbits, finding $t+1$ base cycles which partition the point-set $V$ suffices to confirm the existence of the desired cycle system.

Construction I (for the case $\mathrm{t} \equiv 0,3(\bmod 4))$. Let $\left\{\left(a_{j}, b_{j}\right): 1 \leq j \leq t\right\}$ be a $(t+1)$ ext $\mathcal{S}_{t}$ (as a collection of ordered pairs). For each pair $\left(a_{j}, b_{j}\right)$, form a $2 m$ cycle $\left(x_{1}, \ldots, x_{2 m}\right)$ by joining the points in the set $\left\{m a_{j}-1, \ldots, m\left(a_{j}+1\right)-\right.$ $\left.2 ; m b_{j}-1, \ldots, m\left(b_{j}+1\right)-2\right\}$ like a sort of spiral, i.e., set

$$
x_{2 i-1}=m a_{j}+i-2, x_{2 i}=m\left(b_{j}+1\right)-i-1 \quad \text { for } 1 \leq i \leq m .
$$

Then we have

$$
\begin{aligned}
x_{2 i}-x_{2 i-1} & =m(j+1)-2 i+1 & & \text { for } 1 \leq i \leq m, \\
x_{2 i}-x_{2 i+1} & =m(j+1)-2 i & & \text { for } 1 \leq i \leq m-1, \\
x_{2 m}-x_{1} & =m j . & &
\end{aligned}
$$

It is straightforward to see that the differences from a cycle with (2.1) give every integer in the closed interval $[m(j-1), m(j+1)-1]$ exactly once except $m j$ which is the only one element repeated twice. Note that throughout the paper, we mean a 
"symmetric difference" just by a "difference." That is, a difference $d, 1 \leq d \leq$ $m(t+1)-1$, represents $-d(\bmod 2 m(t+1)-1)$ at the same time.

Besides the $t$ cycles specified by (2.1), take one more $2 m$-cycle $\left(\infty, x_{1}, \ldots, x_{2 m-1}\right)$ by assembling the $2 m$ points of the set $\{0, \ldots, m-2 ; m(t+1)-1, \ldots, m(t+2)-$ $2 ; \infty\}$ in the following manner:

$$
\begin{aligned}
x_{4 i-3} & =m(t+1)+i-2 & & \text { for } 1 \leq i \leq\lfloor(m+1) / 2\rfloor, \\
x_{4 i-2} & =m(t+2)-i-1 & & \text { for } 1 \leq i \leq\lfloor m / 2\rfloor, \\
x_{4 i-1} & =i-1 & & \text { for } 1 \leq i \leq\lfloor m / 2\rfloor, \\
x_{4 i} & =m-i-1 & & \text { for } 1 \leq i \leq\lfloor(m-1) / 2\rfloor .
\end{aligned}
$$

Here $\lfloor\alpha\rfloor$ means the greatest integer not exceeding $\alpha$ (thus including $\alpha$ if $\alpha$ is an integer). Then we have

$$
\begin{aligned}
x_{4 i-2}-x_{4 i-3}=m-2 i+1 & \text { for } 1 \leq i \leq m / 2, \\
x_{4 i-2}-x_{4 i-1}=m t+2 i-1 & \text { for } 1 \leq i \leq m / 2, \\
x_{4 i}-x_{4 i-1}=m-2 i & \text { for } 1 \leq i \leq(m-1) / 2, \\
x_{4 i+1}-x_{4 i}=m t+2 i-2 & \text { for } 1 \leq i \leq(m-1) / 2,
\end{aligned}
$$

which implies that the differences arising from the cycle with (2.2) give every integer in the closed intervals $[1, m-1]$ and $[m t+1, m(t+1)-1]$ exactly once, and $\infty$ twice.

On the other hand, it is easily verified that the $t+1$ cycles specified by (2.1) and (2.2) partition the point-set $V=\{\infty\} \cup \mathbb{Z}_{2 m(t+1)-1}$. Therefore, we can conclude that those $t+1$ cycles can be base cycles for a 1-rotationally resolvable $2 m$-cycle system of $2 K_{2 m(t+1)}$ whenever $t \equiv 0,3(\bmod 4)$. It should be remarked that if $t=0$, i.e., if $v=2 m$, then a single base cycle through $\infty$ satisfying (2.2) generates the desired cycle system in itself.

Example 2.3. The case $m=3$ and $t=3$, i.e., $v=24$. Take the collection of ordered pairs $\{(1,2),(5,7),(3,6)\}$ (equivalently the sequence of integers $(1,1,3,0,2,3,2))$ as a 4 -ext $\mathcal{S}_{3}$. Then the set of base cycles determined by (2.1) and (2.2) for a 1-rotationally resolvable 6-cycle system of $2 K_{24}$ will be as follows:

$$
\{(2,7,3,6,4,5),(14,22,15,21,16,20),(8,19,9,18,10,17),(\infty, 11,13,0,1,12)\}
$$

Construction II (for the case $\mathrm{t} \equiv 1,2(\bmod 4))$. Let $\left\{\left(a_{j}, b_{j}\right): 1 \leq j \leq t\right\}$ be a $t$-ext $\mathcal{S}_{t}$ satisfying $\left(a_{t}, b_{t}\right)=(t+1,2 t+1)$. First, construct base cycles not passing through $\infty$ in the same manner as $(2.1)$ but for $1 \leq j \leq t-1$. Next, make two more base cycles such that the rest of the points $\{0,1, \ldots, m-2 ; m t-1, \ldots, m(t+2)-2$; $m(2 t+1)-1, \ldots, 2 m(t+1)-2 ; \infty\}$ are all used up and the remaining $4 m$ differences $\{1, \ldots, m-1 ; m(t-1)+1, \ldots, m t-1 ; m t, m t, \ldots, m(t+1)-1, m(t+1)-1$; $\infty, \infty\}$ arise from them. The configurations of such two base cycles depend on the value of $m$. 
(i) $m$ is even. Form a base cycle of form $\left(x_{1}, \ldots, x_{2 m}\right)$ on the set of points $\{0, \ldots$, $m-2 ; m t-1, \ldots, m t+m / 2-1 ; m(t+1)+m / 2, \ldots, m(t+2)-2 ; m(2 t+1)+$ $m / 2-2\}$ as follows:

$$
\begin{aligned}
x_{2 i-1} & = \begin{cases}i-1 & \text { for } 1 \leq i \leq m / 2, \\
i-2 & \text { for } m / 2+2 \leq i \leq m,\end{cases} \\
x_{2 i} & = \begin{cases}m(t+2)-i-1 & \text { for } 1 \leq i \leq m / 2-1, \\
m(t+1)-i-1 & \text { for } m / 2 \leq i \leq m,\end{cases} \\
x_{m+1} & =m(2 t+1)+m / 2-2 .
\end{aligned}
$$

The differences from this base cycle give every integer in the closed interval $[m(t-1)+1, m(t+1)-2]$ exactly once and $m(t+1)-1$ twice. Drawing a graph would be of great help to see its systematic structure and we leave it to the reader.

Now, the other base cycle, i.e., the one through $\infty$, should be on the set of points $\{m t+m / 2, \ldots, m(t+1)+m / 2-1 ; m(2 t+1)-1, \ldots, m(2 t+1)+m / 2-$ $3 ; m(2 t+1)+m / 2-1, \ldots, 2 m(t+1)-2 ; \infty\}$ and give the rest of the differences. Here is an explicit configuration for the base cycle of form $\left(\infty, x_{1}, \ldots, x_{2 m-1}\right)$ :

$$
\begin{aligned}
x_{4 i-3} & = \begin{cases}m(t+1)-m / 2+2 i-2 & \text { for } 1 \leq i \leq\lfloor(m+2) / 4\rfloor, \\
m(t+1)+m / 2-2 i+1 & \text { for }\lfloor(m+2) / 4\rfloor+1 \leq i \leq m / 2,\end{cases} \\
x_{4 i-2} & = \begin{cases}m(t+1)+m / 2-2 i+1 & \text { for } 1 \leq i \leq\lfloor(m+2) / 4\rfloor, \\
-3 m / 2+2 i-2 & \text { for }\lfloor(m+2) / 4\rfloor+1 \leq i \leq m / 2,\end{cases} \\
x_{4 i-1} & = \begin{cases}-m / 2+2 i-1 & \text { for } 1 \leq i \leq\lfloor m / 4\rfloor, \\
m / 2-2 i & \text { for }\lfloor m / 4\rfloor+1 \leq i \leq m / 2,\end{cases} \\
x_{4 i} & = \begin{cases}-m / 2-2 i-1 & \text { for } 1 \leq i \leq\lfloor(m+2) / 4\rfloor-1, \\
m(t+1)-m / 2+2 i & \text { for }\lfloor(m+2) / 4\rfloor \leq i \leq m / 2-1 .\end{cases}
\end{aligned}
$$

The differences from the base cycle through $\infty$ supply each integer in the closed intervals $[1, m-1]$ and $[m t, m(t+1)-2]$ exactly once, and $\infty$ twice.

(ii) $m \equiv 1,3(\bmod 6)$. The two required base cycles are on the sets of points $\{0, \ldots, m-2 ; m t-1, \ldots, m t+(m-5) / 2 ; m(t+1)+(m-3) / 2, \ldots, m(t+2)-2 ;$ $2 m(t+1)-2\} \quad$ and $\quad\{m t+(m-3) / 2, \ldots, m(t+1)+(m-5) / 2 ; m(2 t+1)-$ $1, \ldots, 2 m(t+1)-3 ; \infty\}$, respectively. One is of form $\left(x_{1}, \ldots, x_{2 m}\right)$ with

$$
\begin{aligned}
& x_{2 i-1}= \begin{cases}m t+i-2 & \text { for } 1 \leq i \leq(m-1) / 2, \\
m t+3 i-3 & \text { for }(m+1) / 2 \leq i \leq\lfloor(2 m+1) / 3\rfloor, \\
m(t+4)-3 i+1 & \text { for }\lfloor(2 m+1) / 3\rfloor+1 \leq i \leq\lfloor(5 m+3) / 6\rfloor, \\
m(t-1)+3 i-4 & \text { for }\lfloor(5 m+3) / 6\rfloor+1 \leq i \leq m,\end{cases} \\
& x_{2 i}= \begin{cases}m-i-1 & \text { for } 1 \leq i \leq(m-1) / 2, \\
2 m-3 i-1 & \text { for }(m+1) / 2 \leq i \leq\lfloor 2 m / 3\rfloor, \\
-2 m+3 i-2 & \text { for }\lfloor 2 m / 3\rfloor+1 \leq i \leq\lfloor(5 m+1) / 6\rfloor, \\
3 m-3 i & \text { for }\lfloor(5 m+1) / 6\rfloor+1 \leq i \leq m,\end{cases}
\end{aligned}
$$


and the other is of form $\left(\infty, x_{1}, \ldots, x_{2 m-1}\right)$ with

$$
\begin{aligned}
x_{4 i-3} & = \begin{cases}m(t+1)-(m+7) / 2+2 i & \text { for } 1 \leq i \leq\lfloor(m+3) / 4\rfloor, \\
m(t+1)+(m+1) / 2-2 i & \text { for }\lfloor(m+3) / 4\rfloor+1 \leq i \leq(m+1) / 2,\end{cases} \\
x_{4 i-2} & = \begin{cases}m(t+1)+(m-1) / 2-2 i & \text { for } 1 \leq i \leq\lfloor(m+1) / 4\rfloor, \\
-(3 m+3) / 2+2 i & \text { for }\lfloor(m+3) / 4\rfloor+1 \leq i \leq(m-1) / 2,\end{cases} \\
x_{4 i-1} & = \begin{cases}-(m+5) / 2+2 i & \text { for } 1 \leq i \leq\lfloor(m+3) / 4\rfloor-1, \\
m(2 t+1)-1 & \text { for } i=\lfloor(m+3) / 4\rfloor, \\
(m-1) / 2-2 i & \text { for }\lfloor(m+3) / 4\rfloor+1 \leq i \leq(m-1) / 2,\end{cases} \\
x_{4 i} & = \begin{cases}-(m-1) / 2-2 i & \text { for } 1 \leq i \leq\lfloor(m-1) / 4\rfloor-1, \\
m(t+1)-(m+5) / 2+2 i & \text { for }\lfloor(m+1) / 4\rfloor+1 \leq i \leq(m-1) / 2,\end{cases} \\
x_{m+1} & =2 m(t+1)-3 .
\end{aligned}
$$

The differences from the base cycle with (2.3) supply each integer in the closed interval $[m(t-1)+1, m(t+1)-1]$ exactly once except $m(t+1)-2$ which is repeated twice. As for the base cycle with (2.4), each integer in $[1, m-1]$ and $[m t, m(t-1)-3]$ and $m(t+1)-1$ occur precisely once, and $\infty$ twice as the differences from it.

(iii) $m \equiv 5(\bmod 6)$. In this case, we cannot use the configuration $(2.3)$ for the base cycle not passing through $\infty$ without any modification, otherwise the length of the resultant cycle would be less than $2 m$. So, for $(m-3) / 2 \geq 6$ (eventually for $m \geq 17)$, let

$$
\begin{gathered}
x_{2 i-1}= \begin{cases}m t+i-2 & \text { for } 1 \leq i \leq(m-1) / 2, \\
m t+3 i-3 & \text { for }(m+1) / 2 \leq i \leq(2 m-1) / 3-1, \\
m(t+4)-3 i-9 & \text { for }(2 m-1) / 3 \leq i \leq(5 m-1) / 6-3, \\
m(t+4)+3 i+6 & \text { for }(5 m-1) / 6-2 \leq i \leq m-5, \\
m(t+3)-i-9 & \text { for } i=m-4, m-3, \\
m(t+3)-i-4 & \text { for } m-2 \leq i \leq m,\end{cases} \\
x_{2 i}= \begin{cases}m-i-1 & \text { for } 1 \leq i \leq(m-1) / 2, m-4 \leq i \leq m-2, \\
2 m-3 i-1 & \text { for }(m+1) / 2 \leq i \leq(2 m-1) / 3-2, \\
-2 m+3 i+8 & \text { for }(2 m-1) / 3-1 \leq i \leq(5 m-1) / 6-3, \\
3 m-3 i-10 & \text { for }(5 m-1) / 6-2 \leq i \leq m-5, \\
m-i+1 & \text { for } i=m-1, m\end{cases}
\end{gathered}
$$

For the cases when $m=5$ and 11 , below we provide the solutions directly.

$$
\begin{array}{ll}
m=5: \quad & (0,5 t-1,3,5 t, 2,5 t+6,10 t+8,5 t+7,1,5 t+8) \\
m=11: \quad & (0,11 t-1,9,11 t, 8,11 t+1,7,11 t+2,6,11 t+3,5,11 t+15, \\
& 3,11 t+17,4,11 t+16,1,11 t+20,22 t+20,11 t+19,2,11 t+18)
\end{array}
$$


It should be remarked that this modification, including the cases when $m=5$ and 11 , change neither the set of points nor the differences for the cycle. As for the other base cycle, i.e., the one through $\infty,(2.4)$ is valid as it is for any integer $m \geq 5$.

We now know that by letting $v=2 m(t+1)$, the existence problem for 1 rotationally resolvable $2 m$-cycle systems of $2 K_{v}$ can be interpreted as that for the corresponding extended Skolem sequences of order $t$. That is, the existence of a $(t+1)$-ext $\mathcal{S}_{t}$ with $t \equiv 3,0(\bmod 4)$ suffices to prove the existence of a 1-rotationally resolvable $2 m$-cycle system of $2 K_{v}$ for $v \equiv 0,2 m(\bmod 8 m)$, and so does the existence of a $t$-ext $\mathcal{S}_{t}$ with $t \equiv 1,2(\bmod 4)$ satisfying the required condition in Construction II for $v \equiv 4 m, 6 m(\bmod 8 m)$.

\section{EXCEPTIONAL PARAMETERS}

Since the existence of a $(t+1)$-ext $\mathcal{S}_{t}$ with $t \equiv 0,3(\bmod 4)$ implies that of a 1 rotationally resolvable $2 m$-cycle system of $2 K_{v}$ with $v \equiv 2 m, 0(\bmod 8 m)$, Theorem 2.2 and Construction I ensure the following.

Theorem 3.1. There exists a 1-rotationally resolvable $2 m$-cycle system of $2 K_{v}$ whenever $v \equiv 0,2 m(\bmod 8 m)$.

For $v \equiv 4 m, 6 m(\bmod 8 m)$, we can take advantage of the following result due to the authors [9] to complete the sufficiency of Lemma 2.1 except three specific cases.

Theorem $3.2([9]) . \quad$ Whenever $t \equiv 1,2(\bmod 4)$ and $t \geq 6$, there exists a $t$-ext $\mathcal{S}_{t}($ as a collection of ordered pairs) including the pair $(t+1,2 t+1)$.

From Theorem 3.2 and Construction II, we can state the following immediately.

Corollary 3.3. There exists a 1-rotationally resolvable $2 m$-cycle system of $2 K_{v}$ whenever $v \equiv 4 m, 6 m(\bmod 8 m)$ and $v \geq 14 m$.

Unfortunately there does not exist a $t$-ext $\mathcal{S}_{t}$ satisfying the required condition when $t=1,2$, and 5 , which can be easily checked even by hand. This implies that Construction II cannot be applied to the cases $v=4 m, 6 m$, and $12 m$. In the remainder of this section, we will make up for those cases with direct constructions.

Lemma 3.4. When $v=4 m$, 1-rotationally resolvable $2 m$-cycle systems of $2 K_{v}$ exist.

Proof. We just provide the explicit configurations of the required two base cycles and leave the verification to the reader. One of the base cycles is of form $\left(x_{1}, \ldots, x_{2 m}\right)$ with

$$
\begin{aligned}
x_{2 i-1} & = \begin{cases}2 m+2 i-4 & \text { for } 1 \leq i \leq\lfloor m / 2\rfloor+1, \\
4 m-2 i+1 & \text { for }\lfloor m / 2\rfloor+2 \leq i \leq m,\end{cases} \\
x_{2 i} & = \begin{cases}4 m-2 i & \text { for } 1 \leq i \leq\lfloor(m+1) / 2\rfloor, \\
2 m+2 i-3 & \text { for }\lfloor(m+1) / 2\rfloor+1 \leq i \leq m,\end{cases}
\end{aligned}
$$

which is on the set of points $\{2 m-2 ; 2 m, \ldots, 4 m-2\}$. The other is of form $\left(\infty, x_{1}, \ldots, x_{2 m-1}\right)$ and on the set of points $\{0, \ldots, 2 m-3 ; 2 m-1 ; \infty\}$. Its configuration depends on the value of $m$. 
(i) $m \equiv 1(\bmod 4)$.

$$
\begin{array}{rlrl}
x_{4 i-3} & =m+4 i-3 & & \text { for } 1 \leq i \leq(m-1) / 4, \\
x_{4 i-1} & =m+4 i-5 & & \text { for } 1 \leq i \leq(m-1) / 4, \\
x_{2 i} & =m-2 i & & \text { for } 1 \leq i \leq(m-1) / 2 \\
x_{2 i-1} & =3 m-2 i & & \text { for }(m+1) / 2 \leq i \leq m, \\
x_{4 i-2}=-m+4 i-1 & & \text { for }(m+3) / 4 \leq i \leq(m-1) / 2, \\
x_{4 i}=-m+4 i-3 & & \text { for }(m+3) / 4 \leq i \leq(m-1) / 2 .
\end{array}
$$

(ii) $m \equiv 2(\bmod 4)$.

$$
\begin{aligned}
x_{1} & =m-2, & & \\
x_{4 i-3} & =m+4 i-8 & & \text { for } 2 \leq i \leq(m+2) / 4, \\
x_{4 i-1} & =m+4 i-2 & & \text { for } 1 \leq i \leq(m-2) / 4, \\
x_{2 i} & =m-2 i+1 & & \text { for } 1 \leq i \leq m / 2, \\
x_{2 i-1} & =3 m-2 i+1 & & \text { for } m / 2+1 \leq i \leq m, \\
x_{4 i-2} & =-m+4 i-6 & & \text { for }(m+6) / 4 \leq i \leq m / 2, \\
x_{4 i} & =-m+4 i & & \text { for }(m+2) / 4 \leq i \leq m / 2-1 .
\end{aligned}
$$

(iii) $m \equiv 3(\bmod 4)$.

$$
\begin{aligned}
x_{4 i-3}=m-4 i+1 & \text { for } 1 \leq i \leq(m+1) / 4, \\
x_{4 i-1}=m-4 i+3 & \text { for } 1 \leq i \leq(m+1) / 4 \\
x_{2 i}=m+2 i-2 & \text { for } 1 \leq i \leq(m+1) / 2 \\
x_{2 i-1}=-m+2 i-2 & \text { for }(m+3) / 2 \leq i \leq m, \\
x_{4 i-2}=3 m-4 i-1 & \text { for }(m+5) / 4 \leq i \leq(m-1) / 2, \\
x_{4 i}=3 m-4 i+1 & \text { for }(m+5) / 4 \leq i \leq(m-1) / 2 .
\end{aligned}
$$

(iv) $m \equiv 0(\bmod 4)$

$$
\begin{aligned}
x_{1} & =m, & & \\
x_{4 i-3} & =m-4 i+6 & & \text { for } 2 \leq i \leq m / 4+1, \\
x_{4 i-1} & =m-4 i & & \text { for } 1 \leq i \leq m / 4, \\
x_{2 i} & =m+2 i-3 & & \text { for } 1 \leq i \leq m / 2+1, \\
x_{2 i-1} & =-m+2 i-3 & & \text { for } m / 2+2 \leq i \leq m, \\
x_{4 i-2} & =3 m-4 i+4 & & \text { for } m / 4+2 \leq i \leq m / 2, \\
x_{4 i} & =3 m-4 i-2 & & \text { for } m / 4+1 \leq i \leq m / 2-1 .
\end{aligned}
$$

Lemma 3.5. When $v=6 m$, 1-rotationally resolvable $2 m$-cycle systems of $2 K_{v}$ exist. 
Proof. (i) $m \equiv 0(\bmod 2)$. Two of the required base cycles are of form $\left(x_{1}, \ldots, x_{2 m}\right)$, one of which is specified by

$$
\begin{aligned}
x_{2 i-1} & = \begin{cases}m / 2+i-1 & \text { for } 1 \leq i \leq m / 2, \\
5 m / 2-i & \text { for } m / 2+1 \leq i \leq m-1,\end{cases} \\
x_{2 i} & = \begin{cases}5 m / 2-i-1 & \text { for } 1 \leq i \leq m / 2-1, \\
m / 2+i & \text { for } m / 2 \leq i \leq m,\end{cases} \\
x_{2 m-1} & =7 m / 2-1,
\end{aligned}
$$

and the other by

$$
\begin{aligned}
x_{2 i-1} & = \begin{cases}11 m / 2-i-1 & \text { for } 1 \leq i \leq m / 2, \\
7 m / 2+i-2 & \text { for } m / 2+1 \leq i \leq m-1,\end{cases} \\
x_{2 i} & = \begin{cases}7 m / 2+i-1 & \text { for } 1 \leq i \leq m / 2-1, \\
11 m / 2-i-2 & \text { for } m / 2 \leq i \leq m,\end{cases} \\
x_{2 m-1} & =5 m / 2-1 .
\end{aligned}
$$

The two base cycles determined above are on the sets of points $\{m / 2, \ldots$, $5 m / 2-2 ; 7 m / 2-1\}$ and $\{5 m / 2-1 ; 7 m / 2, \ldots, 11 m / 2-2\}$, respectively. It should be noted that they are symmetric with respect to the point $3 m-1$.

Then, the remaining one of form $\left(\infty, x_{1}, \ldots, x_{2 m-1}\right)$ should be on the set of points $\{0, \ldots, m / 2-1 ; 5 m / 2, \ldots, 7 m / 2-2 ; 11 m / 2-1, \ldots, 6 m-2 ; \infty\}$ and we can settle its configuration as follows:

$$
\begin{aligned}
& x_{2 i-1}= \begin{cases}m / 2-i & \text { for } 1 \leq i \leq m / 2, \\
13 m / 2-i-1 & \text { for } m / 2+1 \leq i \leq m,\end{cases} \\
& x_{2 i}=5 m / 2+i-1 \quad \text { for } 1 \leq i \leq m-1 .
\end{aligned}
$$

Note that in this base cycle through $\infty$, any two pairs of points which give an identical difference lie at symmetric positions in the cycle with respect to the point $3 m-1$.

(ii) $m \equiv 1(\bmod 4)$. Two base cycles of form $\left(x_{1}, \ldots, x_{2 m}\right)$ are on the respective sets of points $\{(m-1) / 2, \ldots, 5(m-1) / 2 ;(7 m-3) / 2\} \quad$ and $\quad\{(5 m-1) / 2$; $(7 m+1) / 2, \ldots,(11 m-3) / 2\}$. Their configurations are given by

$$
\begin{aligned}
x_{2 i-1} & =(5 m-3) / 2-i \quad \text { for } 1 \leq i \leq(m+1) / 2, \\
x_{2 i} & =(m-3) / 2+i \quad \text { for } 1 \leq i \leq(m+1) / 2, \\
x_{4 i-3} & =(m-7) / 2+2 i \quad \text { for }(m+7) / 4 \leq i \leq(m-1) / 2, \\
x_{4 i-2} & =(5 m+1) / 2-2 i \text { for }(m+7) / 4 \leq i \leq(m-1) / 2, \\
x_{4 i-1} & =(m-1) / 2+2 i \quad \text { for }(m+3) / 4 \leq i \leq(m-1) / 2, \\
x_{4 i} & =(5 m-5) / 2-2 i \text { for }(m+3) / 4 \leq i \leq(m-3) / 2, \\
x_{2 m-2} & =(3 m-5) / 2, \\
x_{2 m-1} & =(7 m-3) / 2, \\
x_{2 m} & =(3 m-1) / 2,
\end{aligned}
$$


and

$$
\begin{aligned}
& x_{2 i-1}=(7 m-1) / 2+i \quad \text { for } 1 \leq i \leq(m+1) / 2 \text {, } \\
& x_{2 i}=(11 m-1) / 2-i \quad \text { for } 1 \leq i \leq(m+1) / 2, \\
& x_{4 i-3}=(11 m+3) / 2-2 i \text { for }(m+7) / 4 \leq i \leq(m-1) / 2 \text {, } \\
& x_{4 i-2}=(7 m-5) / 2+2 i \quad \text { for }(m+7) / 4 \leq i \leq(m-1) / 2 \text {, } \\
& x_{4 i-1}=(11 m-3) / 2-2 i \text { for }(m+3) / 4 \leq i \leq(m-1) / 2 \text {, } \\
& x_{4 i}=(7 m+1) / 2+2 i \quad \text { for }(m+3) / 4 \leq i \leq(m-3) / 2 \text {, } \\
& x_{2 m-2}=(9 m+1) / 2 \text {, } \\
& x_{2 m-1}=(5 m-1) / 2 \text {, } \\
& x_{2 m}=(9 m-3) / 2 \text {, }
\end{aligned}
$$

respectively. These two base cycles are symmetric with respect to the point $3 m-1$.

As for the one of form $\left(\infty, x_{1}, \ldots, x_{2 m-1}\right)$, it is specified by

$$
\begin{aligned}
& x_{1}=(5 m-3) / 2 \text {, } \\
& x_{2 i-1}=(5 m-3) / 2+i \quad \text { for } 2 \leq i \leq m-1 \text {, } \\
& x_{2 i}= \begin{cases}(m-1) / 2-i & \text { for } 1 \leq i \leq(m-1) / 2, \\
(13 m-3) / 2-i & \text { for }(m+1) / 2 \leq i \leq m-1,\end{cases} \\
& x_{2 m-1}=(7 m-1) / 2
\end{aligned}
$$

on the set of the remaining points $\{0, \ldots,(m-3) / 2 ;(5 m-3) / 2 ;(5 m+1) / 2, \ldots$, $(7 m-5) / 2 ;(7 m-1) / 2 ;(11 m-1) / 2, \ldots, 6 m-2\}$.

(iii) $m \equiv 3(\bmod 4)$. We can use the configuration (3.3) as it is for the base cycle through $\infty$. Concerning the other two base cycles not passing through $\infty$, the configurations differ a little bit from (3.1) and (3.2), but the respective families of differences arising from them remain the same as those from the base cycles with (3.1) and (3.2) in (ii). In this case, the two base cycles of form $\left(x_{1}, \ldots, x_{2 m}\right)$ are specified by

$$
\begin{aligned}
& x_{2 i-1}=(m-3) / 2+i \quad \text { for } 1 \leq i \leq(m-1) / 2 \text {, } \\
& x_{2 i}=(5 m-3) / 2-i \quad \text { for } 1 \leq i \leq(m-1) / 2 \text {, } \\
& x_{4 i-3}=(5 m+1) / 2-2 i \text { for }(m+5) / 4 \leq i \leq(m-1) / 2 \text {, } \\
& x_{4 i-2}=(m-7) / 2+2 i \quad \text { for }(m+5) / 4 \leq i \leq(m-1) / 2 \text {, } \\
& x_{4 i-1}=(5 m-5) / 2-2 i \text { for }(m+1) / 4 \leq i \leq(m-1) / 2 \text {, } \\
& x_{4 i}=(m-1) / 2+2 i \quad \text { for }(m+1) / 4 \leq i \leq(m-3) / 2, \\
& x_{2 m-2}=(3 m-5) / 2 \text {, } \\
& x_{2 m-1}=(7 m-3) / 2 \text {, } \\
& x_{2 m}=(3 m-1) / 2 \text {, }
\end{aligned}
$$


and

$$
\begin{array}{rlrl}
x_{2 i-1} & =(11 m-1) / 2-i & & \text { for } 1 \leq i \leq(m-1) / 2, \\
x_{2 i} & =(7 m-1) / 2+i & & \text { for } 1 \leq i \leq(m-1) / 2, \\
x_{4 i-3} & =(7 m-5) / 2+2 i & & \text { for }(m+5) / 4 \leq i \leq(m-1) / 2, \\
x_{4 i-2} & =(11 m+3) / 2-2 i & & \text { for }(m+5) / 4 \leq i \leq(m-1) / 2, \\
x_{4 i-1} & =(7 m+1) / 2+2 i & & \text { for }(m+1) / 4 \leq i \leq(m-1) / 2, \\
x_{4 i} & =(11 m-3) / 2-2 i & & \text { for }(m+1) / 4 \leq i \leq(m-3) / 2, \\
x_{2 m-2} & =(9 m+1) / 2, & \\
x_{2 m-1} & =(5 m-1) / 2, & \\
x_{2 m} & =(9 m-3) / 2 . &
\end{array}
$$

Lemma 3.6. When $v=12 m$, 1-rotationally resolvable $2 m$-cycle systems of $2 K_{v}$ exist.

Proof. (i) $m \equiv 1(\bmod 2)$. Consider the following 1-ext $\mathcal{S}_{5}$

$$
\left\{\left(a_{j}, b_{j}\right): 1 \leq j \leq 5\right\}=\{(9,10),(4,6),(2,5),(7,11),(3,8)\}
$$

and take four $12 m$-cycles in the same manner as (2.1) for $j=1,3,4$ and 5 . Then we have only to construct two more base cycles by using up the rest of points $\{0, \ldots$, $2 m-2 ; 4 m-1, \ldots, 5 m-2 ; 6 m-1, \ldots, 7 m-2 ; \infty\}$ so that the family of differences arising from them can be $\{1, \ldots, m-1 ; m+1, \ldots, 2 m, 2 m, \ldots, 3 m-1$; $5 m+1, \ldots, 6 m-1 ; \infty, \infty\}$. Here are the solutions: the one of form $\left(x_{1}, \ldots, x_{2 m}\right)$ is on the set of points $\{(3 m-1) / 2, \ldots, 2 m-2 ; 4 m-1, \ldots, 5 m-2 ; 6 m-1, \ldots$, $(13 m-3) / 2\}$ and satisfies

$$
\begin{aligned}
& x_{2 i-1}=4 m+i-2 \quad \text { for } 1 \leq i \leq m \text {, } \\
& x_{2 i}= \begin{cases}2 m-i-1 & \text { for } 1 \leq i \leq(m-1) / 2, \\
7 m-i-1 & \text { for }(m+1) / 2 \leq i \leq m,\end{cases}
\end{aligned}
$$

and the other of form $\left(\infty, x_{1}, \ldots, x_{2 m-1}\right)$ is on the set of points $\{0, \ldots, 3(m-1) / 2$; $(13 m-1) / 2, \ldots, 7 m-2 ; \infty\}$ and satisfies

$$
\begin{aligned}
x_{2 i-1} & =(m-3) / 2+i \\
x_{2 i} & = \begin{cases}(m-1) / 2-i & \text { for } 1 \leq i \leq m, \\
(15 m-3) / 2-i & \text { for } 1 \leq i \leq(m-1) / 2\end{cases}
\end{aligned}
$$

(ii) $m \equiv 0(\bmod 2)$. First, by using (2.1) with (3.4), form the $2 m$-cycles through $\infty$ for $j=1,4$ and 5. And then, in the two cycles for $j=4$ and 5, replace $x_{1}=7 m-1$ with $4 m-1$, and $x_{m+1}=7 m / 2-1$ with $3 m / 2-1$, respectively. To be precise, set

$$
\begin{aligned}
x_{1} & =4 m-1, \\
x_{2 i-1} & =7 m+i-2 \quad \text { for } 2 \leq i \leq m, \\
x_{2 i} & =12 m-i-1 \quad \text { for } 1 \leq i \leq m
\end{aligned}
$$


as the modified configuration corresponding to the case $j=4$ and

$$
\begin{aligned}
x_{2 i-1} & =3 m+i-2 \quad \text { for } 1 \leq i \leq m, i \neq m / 2+1, \\
x_{2 i} & =9 m-i-1 \quad \text { for } 1 \leq i \leq m, \\
x_{m+1} & =3 m / 2-1
\end{aligned}
$$

as the one corresponding to the case $j=5$. Note that these modifications preserve the differences arising from the original cycles.

Next, take two base cycles of form $\left(x_{1}, \ldots, x_{2 m}\right)$ on the sets of points $\{2 m, \ldots$, $3 m-2 ; 7 m / 2-1 ; 5 m, \ldots, 6 m-1\}$ and $\{3 m / 2, \ldots, 2 m-1 ; 4 m, \ldots, 5 m-1 ; 6 m, \ldots$, $13 m / 2-1\}$ according to the configurations

$$
\begin{aligned}
& x_{2 i-1}= \begin{cases}2 m+i-1 & \text { for } 1 \leq i \leq m / 2, \\
2 m+i-2 & \text { for } m / 2+2 \leq i \leq m,\end{cases} \\
& x_{2 i}=6 m-i \quad \text { for } 1 \leq i \leq m, \\
& x_{m+1}=7 m / 2-1
\end{aligned}
$$

for one and

$$
\begin{aligned}
& x_{2 i-1}=4 m+i-1 \quad \text { for } 1 \leq i \leq m \text {, } \\
& x_{2 i}= \begin{cases}2 m-i & \text { for } 1 \leq i \leq m / 2, \\
7 m-i & \text { for } m / 2+1 \leq i \leq m\end{cases}
\end{aligned}
$$

for the other. Now it turns out that we have only to find one more base cycle passing through $\infty$ on the set of points $\{0, \ldots, 3 m / 2-2 ; 13 m / 2, \ldots, 7 m-1 ; \infty\}$. We will look at this by dividing the present case between $m \equiv 0,4(\bmod 6)$ and $m \equiv 2(\bmod 6)$. Below the configuration of the desired base cycle of form $(\infty$, $\left.x_{1}, \ldots, x_{2 m-1}\right)$ is indicated for each subcase.

(ii) $m \equiv 0,4(\bmod 6)$.

$$
\begin{aligned}
& x_{2 i-1}= \begin{cases}m / 2+3 i-2 & \text { for } 1 \leq i \leq\lfloor(m+2) / 6\rfloor \\
3 m / 2-3 i+2 & \text { for }\lfloor(m+2) / 6\rfloor+1 \leq i \leq\lfloor(m+2) / 3\rfloor, \\
-m / 2+3 i-3 & \text { for }\lfloor(m+2) / 3\rfloor+1 \leq i \leq m / 2, \\
15 m / 2-i & \text { for } m / 2+1 \leq i \leq m,\end{cases} \\
& x_{2 i}= \begin{cases}m / 2-3 i & \text { for } 1 \leq i \leq\lfloor m / 6\rfloor, \\
-m / 2+3 i-1 & \text { for }\lfloor m / 6\rfloor+1 \leq i \leq\lfloor m / 3\rfloor, \\
3 m / 2-3 i+1 & \text { for }\lfloor m / 3\rfloor+1 \leq i \leq m / 2, \\
m / 2+i-1 & \text { for } m / 2+1 \leq i \leq m-1\end{cases}
\end{aligned}
$$

(ii) $)^{\prime \prime} m \equiv 2(\bmod 6)$.

$$
\begin{gathered}
x_{2 i-1}= \begin{cases}m / 2-3 i+2 & \text { for } 1 \leq i \leq(m+4) / 6, \\
-m / 2+3 i-3 & \text { for }(m+10) / 6 \leq i \leq m / 2, \\
15 m / 2-i & \text { for } m / 2+1 \leq i \leq m,\end{cases} \\
x_{2 i}= \begin{cases}m / 2+3 i-1 & \text { for } 1 \leq i \leq(m-2) / 6, \\
3 m / 2-3 i+1 & \text { for }(m+4) / 6 \leq i \leq m / 2, \\
m / 2+i-1 & \text { for } m / 2+1 \leq i \leq m-1\end{cases}
\end{gathered}
$$


Example 3.7. The case $m=4$ and $t=6$, i.e., $v=48$. According to the proof of Lemma 3.6 (ii) and (ii)', the set of base cycles for a 1-rotationally resolvable 8-cycle system of $2 K_{48}$ will be given as follows:

$$
\begin{aligned}
& \{(35,42,36,41,37,40,38,39),(15,46,28,45,29,44,30,43) \\
& (11,34,12,33,5,32,14,31),(8,23,9,22,13,21,10,20) \\
& (16,7,17,6,18,25,19,24),(\infty, 3,0,2,1,27,4,26)\}
\end{aligned}
$$

For the case $\lambda=2$, the sufficiency of Lemma 2.1 is assured by Theorem 3.1, Corollary 3.3, Lemmas 3.4, 3.5 and 3.6. Therefore, the main theorem is finally established.

Theorem 3.8. There exists a 1-rotationally resolvable $2 m$-cycle system of $\lambda K_{v}$ if and only if $\lambda$ is even and $v \equiv 0(\bmod 2 m)$.

Since Lemma 2.1 describes nothing but necessary conditions for the existence of resolvable even-cycle systems, Theorem 3.3 eventually asserts the following.

Corollary 3.9. There exists a resolvable $2 m$-cycle system of $\lambda K_{v}$ if and only if $\lambda$ is even and $v \equiv 0(\bmod 2 m)$.

As mentioned in Section 1, there are several interesting results on cycle systems, some of which are by virtue of Skolem sequences. But as far as the authors know, the existence problem of resolvable odd-cycle systems with a rotational automorphism is still open.

\section{ACKNOWLEDGMENTS}

The authors thank referees for their helpful comments to improve the readability of the paper.

\section{REFERENCES}

[1] B. Alspach, The Oberwolfach problem, The CRC handbook of combinatorial designs, C. J. Colbourn and J. H. Dinitz (Editors), CRC Press, Boca Raton, FL, 1996, pp. 394-395.

[2] B. Alspach, P. J. Schellenberg, D. R. Stinson, and D. Wagner, The Oberwolfach problem and factors of uniform odd length cycles, J Combin Theory Ser A 52 (1989), 20-43.

[3] C. A. Baker, Extended Skolem sequences, J Combin Des 3 (1995), 363-379.

[4] M. Buratti, Existence of 1-rotational $k$-cycle systems of the complete graph, Graphs Combin, (in press).

[5] M. Buratti, 1-Rotational Steiner triple systems over arbitrary groups, J Combin Des 9 (2001), 215-226.

[6] M. Buratti, Rotational $k$-cycle systems of order $v<3 k$; another proof of the existence of odd cycle systems, J Combin Des, 11(2003), 433-444, this issue.

[7] M. Buratti and A. Del Fra, Existence of cyclic $k$-cycle systems of the complete graph, Discrete Math 261 (2003), 113-125.

[8] C. J. Colbourn and A. Rosa, Triple systems, Oxford University Press, New York, 1999. 
[9] H.-L. Fu and M. Mishima, 1-Rotationally resolvable 4-cycle systems of $2 K_{v}$, J Combin Des 10 (2002), 116-125.

[10] P. Gvozdjak, On the Oberwolfach problem for complete multigraphs, Discrete Math 173 (1997), 61-69.

[11] D. G. Hoffman and P. J. Shellenberg, The existence of $C_{k}$-factorizations of $K_{2 n}-F$, Discrete Math 97 (1991), 243-250.

[12] E. Köhler, Das Oberwolfacher problem, Mitt Math Ges Humburg 10 (1973), 52-57.

[13] C. C. Lindner and C. A. Rodger, Decomposition into cycles II: Cycle systems, Contemporary design theory: A collection of surveys, J. H. Dinitz and D. R. Stinson (Editors), John Wiley \& Sons, New York, 1992, pp. 325-369.

[14] R. Rees and N. Shalaby, Simple and indecomposable twofold cyclic triple systems from Skolem sequences, J Combin Des 8 (2000), 402-410.

[15] C. A. Rodger, Cycle systems, The CRC handbook of combinatorial designs, C. J. Colbourn and J. H. Dinitz (Editors), CRC Press, Boca Raton, FL, 1996, pp. 266-270.

[16] A. Rosa, Poznámka o cyklikých Steinerových systémoch torojíc, Mat Fyz Časopis 16 (1966), 285-290.

[17] N. Shalaby, Skolem sequences, The CRC handbook of combinatorial designs, C. J. Colbourn and J. H. Dinitz (Editors), CRC Press, Boca Raton, FL, 1996, pp. 457-461. 